\title{
A polarization encoded photon-to-spin interface
}

\author{
K. C. Chen $\left(\mathbb{D}^{1,2 凶}\right.$, E. Bersin $\left(\mathbb{D}^{1,2}\right.$ and D. Englund $\left(\mathbb{D}^{1}\right.$
}

We propose an integrated photonics device for mapping qubits encoded in the polarization of a photon onto the spin state of a solid-state defect coupled to a photonic crystal cavity: a "polarization-encoded photon-to-spin interface" (PEPSI). We perform a theoretical analysis of the state fidelity's dependence on the device's polarization extinction ratio and atom-cavity cooperativity. Furthermore, we explore the rate-fidelity trade-off through analytical and numerical models. In simulation, we show that our design enables efficient, high fidelity photon-to-spin mapping.

npj Quantum Information (2021)7:2; https://doi.org/10.1038/s41534-020-00337-3

\section{INTRODUCTION}

Quantum networks are being developed to distribute entanglement across distant nodes. A central requirement common to most quantum network approaches is the development of efficient interfaces between spins and photonic qubits ${ }^{1}$. Among the various photonic degrees of freedom, encoding in the polarization basis $\{|H\rangle,|V\rangle\}$ (horizontal and vertical) is attractive over number-state encoding because photon loss becomes a heralded error ${ }^{2}$.

To couple the polarization-encoded photon qubit $\left|\psi_{P}\right\rangle=a|H\rangle+$ $\beta|V\rangle$ to an atomic memory in a network node, Duan and Kimble ${ }^{3}$ proposed the scheme illustrated in Fig. 1a. The incoming photon passes through a polarizing beam splitter (PBS) so that only the $V$ polarization is reflected off a single-sided cavity whose mode couples with the $|\downarrow\rangle \leftrightarrow\left|\downarrow^{\prime}\right\rangle$ transition. The $H$ polarization is reflected off a mirror, and recombines with the $V$ polarization to form an entangled spin-photon state: $\left|\psi_{\text {ent.out }}\right\rangle=-a|H, \downarrow\rangle+$ $\beta|V, \downarrow\rangle-a|H, \uparrow\rangle-\beta|V, \uparrow\rangle$. Subsequent measurement of the photonic state heralds the transfer of the polarization qubit to the atom, as demonstrated in recent experiments using trapped neutral atoms ${ }^{4}$ and diamond color centers ${ }^{5}$.

However, in a free-space setup, a major technical challenge concerns the need to maintain stability of the phase difference between two spatially separated meters-long polarization paths ${ }^{4}$. In this paper, we propose a monolithic, micron-scale photonic structure that combines the $H$ and $V$ paths into one phase-stable architecture (Fig. 1b). We estimate that this system will enable state transfer fidelity exceeding $99 \%$. This polarization-encoded photon-to-spin interface (PEPSI) greatly simplifies quantum networking with polarization-encoded photons coupled to atomic memories.

\section{RESULTS}

Device

Qubits based on single atoms such as solid-state color centers, neutral atoms, and trapped ions have emerged as promising systems for quantum networking applications. In particular, group-IV color centers in diamond are attractive candidates due to their excellent optical and spin coherence ${ }^{6-9}$. In this paper, we focus on the negatively charged silicon-vacancy center (SiV) coupled to a diamond nanocavity, though the approach generalizes to other stationary qubits such as neutral atoms ${ }^{4}$, trapped ions ${ }^{10,11}$, and quantum dots ${ }^{12,13}$.

As illustrated in Fig. 1b, the structure comprises (i) a polarization-dependent reflector (PDR) for the $H$ (TE) mode and (ii) an over-coupled single-sided cavity for the $V$ (TM) mode. The PEPSI collapses both interferometric arms into one co-propagating path that greatly suppresses phase instability stemming from environmental noise. In contrast, bulk optics suffer from thermal and vibrational fluctuations that incur phase noise, which requires phase stabilization costly in operation time ${ }^{5}$.

The PDR shown in Fig. 1c uses a corrugated photonic crystal design with periodicity $a=184 \mathrm{~nm}$, width $W=2.07 a$, modulation amplitude $\mathrm{d} W=3.97 a$. An adiabatic taper transfers photons to a 1D photonic crystal nanocavity coupled to the SiV. The remainder of this paper analyzes the performance this phase-stable device. Specifically, we investigate the impact of PEPSI device parameters on state transfer fidelity (see "Effects of device imperfections"), the rate-fidelity trade-off in a quantum network link (see "Quantum state transfer rate"), and extensions to a scalable photonic integrated circuit (PIC) platform (see "Discussion").

Effects of device imperfections

To analyze the state transfer process, we consider a single photon $\left(\left|\psi_{P}\right\rangle\right)$ incident on a cavity-coupled spin qubit $\left|\psi_{s, i}\right\rangle=(|\uparrow\rangle+|\downarrow\rangle) / \sqrt{2}$. Using a Schrödinger picture evolution, we calculate the resulting spin state $\left|\psi_{s, f}(i)\right\rangle$ after detection of the reflected photon in the polarization diagonal basis $\{|D\rangle,|A\rangle\}$. Its overlap with the desired state transferred from the polarization qubit defines the state fidelity $\mathcal{F}$, of which we take the average ${ }^{14}$ :

$\mathcal{F}=\frac{1}{4} \sum_{i} \mathcal{F}_{i}=\frac{1}{4} \sum_{i}\left|\left\langle\phi_{i} \mid \psi_{s, f}(i)\right\rangle\right|^{2}$,

where $\left|\phi_{1,2}\right\rangle=(|\downarrow\rangle \pm|\uparrow\rangle) / \sqrt{2},\left|\phi_{3,4}\right\rangle=(|\downarrow\rangle \pm \mathrm{i}|\uparrow\rangle) / \sqrt{2}$ (see Supplementary Note 2).

When a device is perfect such that a lossless PDR has infinite polarization extinction ratios and a nanocavity has perfect waveguide-cavity coupling $\left(\kappa_{\mathrm{wg}} / K=1\right)$, the cavity reflection solely determines the fidelity that scales as $(C-1) /(C+1)$ in the large cooperativity limit ${ }^{4}$ (see "Methods" and Supplementary Note 3 ). However, when the PDR has finite extinction ratios and scattering losses, and the single-sided cavity has a reduced waveguide-cavity coupling efficiency $\kappa_{\mathrm{wg}} / K<1$, the need to

\footnotetext{
${ }^{1}$ Research Laboratory of Electronics, Massachusetts Institute of Technology, Cambridge, MA 02139, USA. ${ }^{2}$ These authors contributed equally: K. C. Chen, E. Bersin.

凶email: kcchen@mit.edu
} 
balance losses becomes especially important to achieving high fidelity. For example, considering the desired state $\left|\phi_{1}\right\rangle$ where $a=\beta=1$, balancing losses entails matching the two coefficients $\left|r_{H, \text { on }}-r_{V, \text { on }}\right|=\left|r_{H, \text { off }}+r_{V, \text { off }}\right|$, which are both functions of PDR transmissivity/reflectivity and the cavity reflectivity (see "Methods").

Figure 2 a shows $\mathcal{F}$ as a function of the PDR's width and modulation amplitude given a low scattering loss and fixed cavity parameters corresponding to our design: waveguide-cavity coupling $\kappa_{\mathrm{wg}} / \kappa \approx 0.83$ and cooperativity $C=100$, which has already been experimentally demonstrated in ref. ${ }^{5}$. We find the upper bound of the fidelity is maximized at $99.978 \%$ (assuming perfect gate and detection fidelities) when $W=380 \mathrm{~nm}$ and $\mathrm{d} W=$ $730 \mathrm{~nm}$, corresponding to transmission and reflection extinction ratios of 17.72 and $18.93 \mathrm{~dB}$ for 20 periodicities. In order to

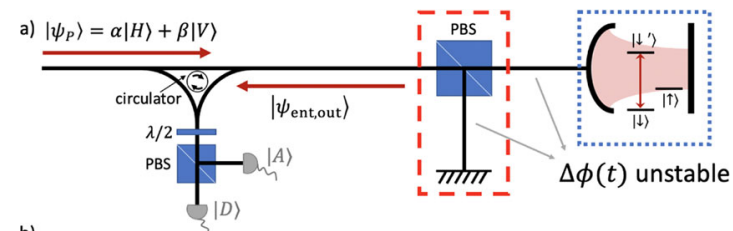

b)

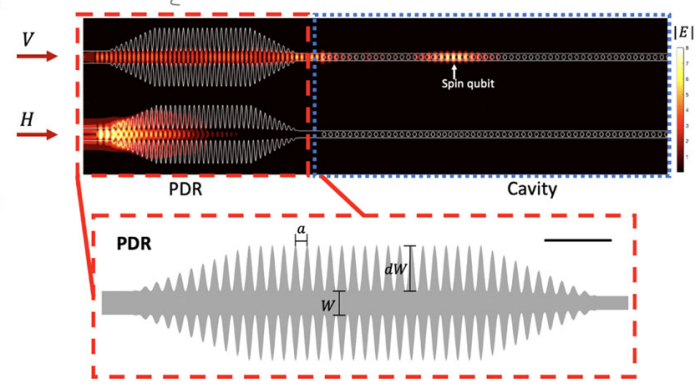

Fig. 1 Polarization-encoded photon-to-spin interface. a The Duan-Kimble scheme for polarization-spin mapping. The requirements of a high extinction polarizing beam splitter (PBS), strong cavity-atom coupling, and stabilization of the phase mismatch between arms $\Delta \phi(t)$ all make implementation with bulk optics challenging. b Our proposal for a phase-stable monolithic device (PEPSI) that implements the protocol in a. A polarization-dependent reflector (PDR, red dashed lines) then reflects $H$ light while passing $V$ light through to interact with the cavity-emitter system (blue dashed lines). c A zoom-in depiction of the PDR with geometry parameters a (periodicity), $W$ (width), and $\mathrm{d} W$ (modulation amplitude). The scale bar corresponds to $1 \mu \mathrm{m}$. controllably balance losses to maximize the state transfer fidelity, we also include an $\mathrm{H}$ attenuator that only diminishes the incoming $H$ light (see Supplemental Note 1). For each set of parameter values $\{\mathrm{d} W, W\}$, we calculate the $H$ attenuation factor $\eta_{H}$ that maximizes $\mathcal{F}$ in Fig. 2b. For the particular design with $W=380 \mathrm{~nm}$ and $\mathrm{d} W=730 \mathrm{~nm}$, the optimal upper bound $\mathcal{F}=99.978 \%$ is reached when $\eta_{H}=66 \%$. In fact, by tuning $\eta_{H}$ appropriately, we observe that $\mathcal{F}$ well exceeds $99 \%$ for any dW between 700 and $750 \mathrm{~nm}$ and similarly for any $W$ between 360 and $420 \mathrm{~nm}$, providing the PEPSI tolerance to fabrication errors.

Additionally, the state transfer fidelity intimately relates to the cavity parameters. Figure $2 \mathrm{c}$ shows that the state fidelity monotonically improves with the atom-cavity cooperativity since the cavity reflectivity $r \propto(C-1) /(C+1)$ approaches +1 . For our device, the atom-cavity cooperativity of $C=100$ gives $\mathcal{F}=99.978 \%$. We also analyze the fidelity's dependence on the waveguide-cavity coupling strength. As a result of balanced losses, Fig. $2 d$ indicates that the fidelity is maximized when $\kappa_{\mathrm{wg}} / K=0.82$ and decreases as the waveguide-cavity coupling deviates from the optimal point. An under-coupled or critically coupled cavity would result in a severely degraded state transfer due to insufficient cavity reflection. On the other hand, overcoupling past $\kappa_{\mathrm{wg}} / K=0.82$ would curtail the atom-cavity interaction and consequently lowers the fidelity. However, our calculation shows that the fidelity can still exceed $99 \%$ as long as $\kappa_{w g} / K$ falls between 0.73 and 0.93, granting again partial immunity to fabrication errors.

\section{Quantum state transfer rate}

We now analyze the performance of the PEPSI in facilitating quantum state transfer across a lossy network link by looking at the rate-fidelity trade-off for a device realized in simulation, parameterized in the section "Device". We denote the probability of a photon entering and returning through the PDR as $\eta_{H} \eta_{\mathrm{PDR}}^{2} R_{\text {cav }}$, where $R_{\text {cav }}$ is the cavity reflection coefficient and $1-R_{\text {cav }}$ is the cavity decay rate into the environment (assume negligible transmission through the single-sided cavity). $\eta_{\mathrm{PDR}}$ is the transmission efficiency of the PDR. As shown in Fig. 3a, the protocol begins by initializing the spin qubit in a superposition state $(|\uparrow\rangle+|\downarrow\rangle) / \sqrt{2}$ in a time $\tau_{\text {reset }}=30 \mu \mathrm{s}$ as demonstrated in ref. ${ }^{5}$. A photonic qubit $\left|\psi_{P}\right\rangle$ launched across the link with transmissivity $\eta_{\text {link }}$ reaches the PDR shown in Fig. $3 \mathrm{~b}$. If the reflected photon is detected as described above with probability $p_{\text {det }}=\eta_{\text {link }} \eta_{H} \eta_{\mathrm{PDR}}^{2} R_{\text {cav }} \eta_{\text {det }}$, the spin qubit is projected to the state
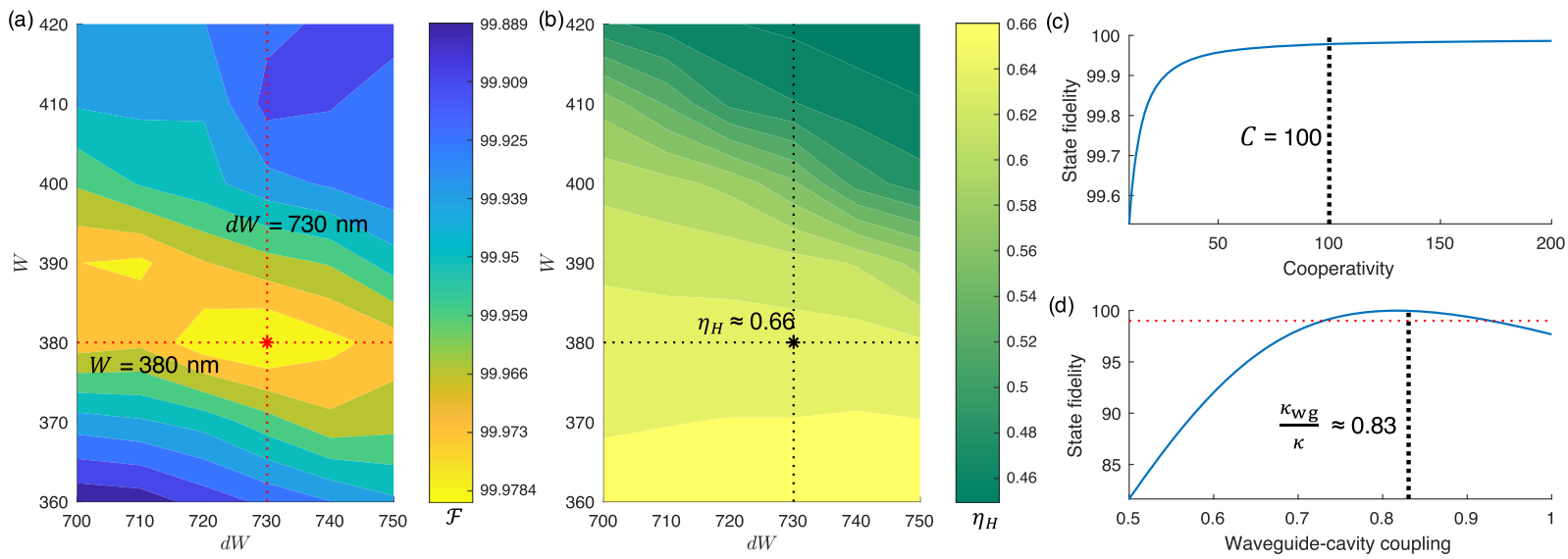

Fig. 2 Fidelity as a function of device imperfections. a $\mathcal{F}$ is plotted as a function of the two PDR geometry parameters: the width $W$ and the modulation amplitude $\mathrm{d} W . W=380 \mathrm{~nm}$ and $\mathrm{d} W=730 \mathrm{~nm}$ are chosen for our particular device with an optimum $\mathcal{F}=99.978 \%$. b The optimal $H$ polarization attenuation factor for balancing losses as a function of $W$ and $d W$. c, d The state fidelity as functions of the atom-cavity cooperativity and waveguide-cavity coupling. The atom-cavity cooperativity is calculated using Eq. (15). The PDR is designed specifically for a single-sided cavity with $C=100$. The waveguide-cavity coupling is tuned to $\kappa_{\mathrm{wg}} / \kappa \approx 0.83$ to maximize fidelity within the allowable design space. The red dashed line indicates the threshold $\mathcal{F}=99 \%$, which is satisfied in the range $\kappa_{\text {wg }} / \kappa=[0.73,0.93]$. 

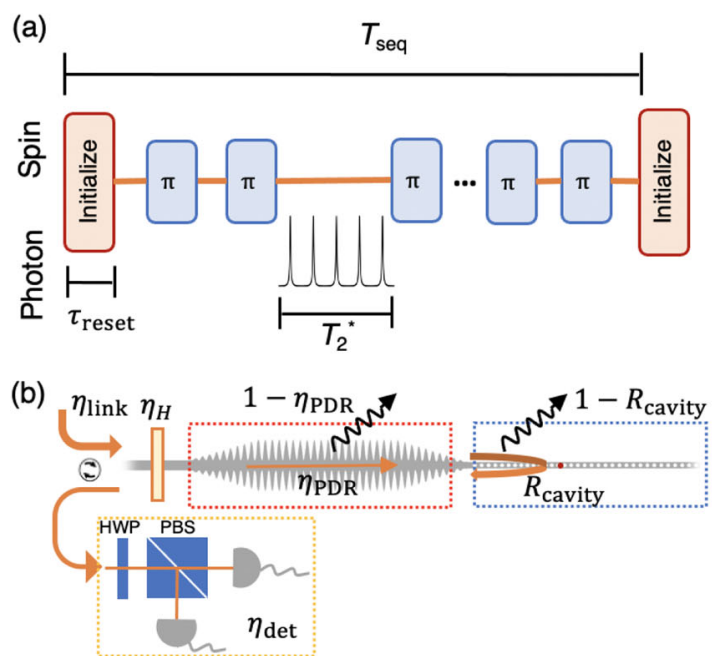

(c)

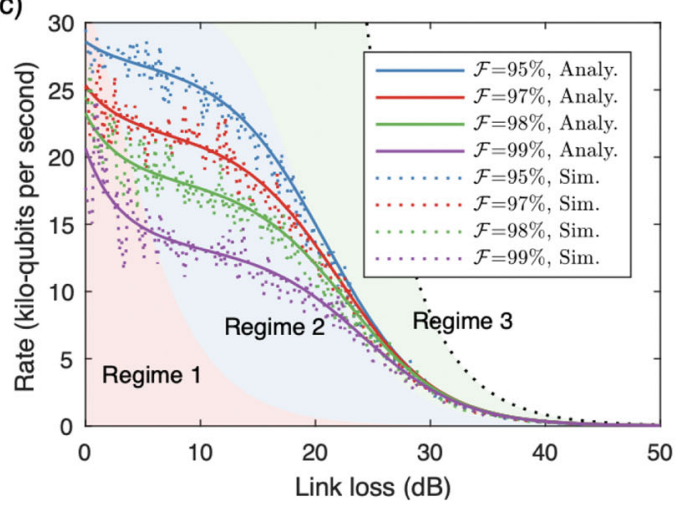

Fig. 3 Rate-fidelity trade-off as a function of link loss. a Pulse sequences for conducting quantum state transfer between a polarization qubit and a spin qubit. b A diagram depicting where scattering losses occur. c Transfer rate (kilo-qubits per second, operated at $5.81 \mathrm{MHz}$ clock rate) as a function of link loss $1-\eta_{\text {link }}$ for four fidelity constraints: $\mathcal{F}=95,97,98$, and $99 \%$. We plot both the analytical solutions (solid) and the Monte Carlo simulations (dashed). We categorize the rate as a function of link loss into Regime 1 (red), Regime 2 (blue), and Regime 3 (green). The black dashed line denotes the repeaterless bound for quantum key distribution protocols ${ }^{15}$.

$a|\downarrow\rangle+\beta|\uparrow\rangle$ (see "Methods") with fidelity given by Eq. (1). If no photon is detected, the protocol is repeated.

However, when $\eta_{\text {link }} \ll 1$, most transmission attempts do not interact with the spin, and time can be saved by not re-initializing on every transmission attempt. In particular, we consider a series of $N$ photons injected into the link after spin re-initialization. These photons are interspersed by dynamical decoupling pulses $(\pi-$ pulses in Fig. 3a) to maintain memory coherence. If the detector registers a click for the $m$ th attempt, the receiver blocks the subsequent $N-m$ pulses. The complication is that any photon that reaches the cavity but is subsequently lost produces an unheralded error with probability $p_{\mathrm{e}}=\eta_{\text {link }} \eta_{H} \eta_{\mathrm{PDR}}\left(1-R_{\text {cav }}\right)$, since the environment projects the spin to a mixed state $\rho_{\text {mixed }}=\frac{1}{2} \mathbb{I}$. Thus, the optimum fidelity for a given device is achieved by reinitializing the spin qubit in advance of every photon transmission. However, the photon can also be either lost in the link before reaching the spin with probability $p_{\text {lost }}=1-p_{\mathrm{e}}-p_{\text {det }}$ or heralded by the detector with probability $p_{\text {det }}$. Conditioned on not detecting a click, the probability of photon loss without contaminating the spin is $p_{\text {lost }} /\left(1-p_{\text {det }}\right)$, and therefore the probability of photon never reaching the spin after $m-1 \leq N$ channel uses is $\left(p_{\text {lost }} /\left(1-p_{\text {det }}\right)\right)^{m-1}$.
Given a detector click on the $m$ th attempt, the probability that at least one error has occurred in the preceding $m-1$ bins is

$P_{\text {error }}(m)=1-\left(\frac{p_{\text {lost }}}{1-p_{\text {det }}}\right)^{m-1}$.

The average error probability is found by summing over all possible sequences up to a total of $N$ attempts, each sequence weighted by $P(m$ th click ), the probability of detecting a click on the $m$ th attempt:

$$
\begin{aligned}
P_{\text {error }} & =\sum_{m=1}^{N} P_{\text {error }}(m) P\left(m^{\text {th }} \text { click }\right) \\
& =1-\left(1-p_{\text {det }}\right)^{N}-p_{\text {det }} \frac{1-p_{\text {lost }}^{N}}{1-p_{\text {lost }}}
\end{aligned} .
$$

The average state fidelity after the protocol that uses sequences of length $N$ before resetting the memory is then:

$\mathcal{F}=\left\langle\psi_{\text {ideal }}\left|\left(1-P_{\text {error }}\right) \rho_{0, \text { eff }}+P_{\text {error }} \rho_{\text {mixed }}\right| \psi_{\text {ideal }}\right\rangle$,

where $\left|\psi_{\text {ideal }}\right\rangle$ is the ideal transferred quantum state and $\rho_{0, \text { eff }}$ is the effective density matrix incorporating device imperfections and detection error (see "Methods"). We can solve Eq. (4) for the maximum number of channel uses before spin re-initialization $N_{\max }$ under a given fidelity constraint, e.g. $\mathcal{F}=99 \%$.

Each sequence (duration $T_{\text {seq }}$ as denoted in Fig. 3a) of $N_{\max }$ transmission attempts has a probability $P_{\text {success }}=1-$ $\left(1-p_{\text {det }}\right)^{N_{\max }}$ to detect at least one click. The number of failed sequences (i.e. each sequence of $N_{\max }$ attempts without clicks followed by a memory reset) before a successful one is given by the geometric distribution. Thus, the average time of failed sequences per detector click is

$T_{\text {failures }}=\frac{\left(1-p_{\text {det }}\right)^{N_{\max }}}{1-\left(1-p_{\text {det }}\right)^{N_{\max }}}\left[N_{\text {max }} \tau_{\text {pulse }}+\tau_{\text {reset }}\right]$,

where $4 \tau_{\text {pulse }}$ is an effective pulse time accounting for repetition rate and dynamical decoupling $\pi$ pulses (see Supplemental Note 4). After these failures, there is a successful sequence where the $m$ th bin yields a click, which takes an average time of:

$$
\begin{aligned}
T_{\text {success }} & =\tau_{\text {reset }}+\sum_{m=1}^{N_{\text {max }}} P\left(m^{\text {th }} \text { click }\right) \mathrm{m} \tau_{\text {pulse }} \\
& =\tau_{\text {reset }}+\tau_{\text {pulse }}\left(\frac{P_{\text {success }}}{P_{\text {det }}}-N_{\max }\left(1-p_{\text {det }}\right)^{N_{\text {max }}}\right)
\end{aligned}
$$

The average quantum state transfer rate is then the inverse of the time per success:

$\bar{\Gamma}=\frac{1}{T_{\text {failures }}+T_{\text {success }}}$.

In Fig. 3c, we explore the trade-off between the heralded state fidelity $\mathcal{F}$ and the average rate accounting for both polarizations (see "Methods"). We verify our analytical solutions with Monte Carlo simulations, and show that the PEPSI can achieve transfer rate exceeding 1 kilo-qubits per second even at high link loss $\sim 30 \mathrm{~dB}$.

We divide the rate into three regimes. In Regime 1 (shaded red) where $N_{\text {max }}$ is low, high-fidelity state transfer prohibits increasing $N_{\max }$ to offset losses in the channel, causing an exponential rate loss that intensifies for higher fidelity constraint, e.g. $\mathcal{F}=99 \%$. On the other hand, for a more relaxed fidelity constraint, e.g. $\mathcal{F}=95 \%$, the spin does not need to be re-initialized as frequently and the rate does not fall off as drastically.

As the link loss increases in Regime 2 (shaded blue), the number of transmission attempts per memory reset also increases. However, the time per success is still dominated by memory reset time in this regime where $\tau_{\text {reset }}>N_{\text {max }} \tau_{\text {pulse. }}$. As a result, the rate of increase for the number of sequences prior to detecting a click stays constant, and the rate consequently remains relatively flat. 
However, in Regime 3 (shaded green) when the number of transmission attempts per sequence increases such that $N_{\text {max }} \tau_{\text {pulse }}>$ $\tau_{\text {reset }} \eta_{\text {link }}$ becomes the rate-limiting factor. In this regime, the rate thus approaches the channel-limited bound (black dashed) given by $\propto \eta_{\text {link }} / \tau_{\text {pulse }}{ }^{15}$.

\section{DISCUSSION}

Practical quantum repeater nodes will likely require multiplexing over a large number of qubits. To this end, we consider the PEPSI PIC illustrated in Fig. 4. An incoming photonic qubit $\left|\psi_{P}\right\rangle$ enters through a PDR followed by a Mach-Zehnder interferometer (MZI) tree network, which routes the photon to a quantum memory. The $\mathrm{PIC}$ with $>\mathrm{GHz}$ modulation of the $\mathrm{MZIs}^{16}$ can perform state mapping across the memory array simultaneously by sending multiplexed photons to different atom-coupled cavities. As a result, the transfer rate improves by a factor of $N_{\text {cav }}$ equivalent to the number of memories connected to the tree network. The architecture can also produce heralded entanglement by sending a photon that enters an MZI 50:50 beam splitter immediately before entering any two neighboring memories. Repeated heralding then produces a cluster of entangled nodes useful for quantum key distribution protocols.

Furthermore, an active PIC provides tunability essential for efficient quantum state transfer. For example, aluminum nitride photonic circuits have integrated 128 diamond waveguidecoupled color centers ${ }^{17}$ and can enable piezoelectric spectral tuning of photonic crystal cavities ${ }^{18}$ and diamond color center emission ${ }^{19,20}$. Integration by pick-and-placing allows postselecting high-performing devices, and additional on-chip waveplates and polarizers in conjunction with the PDR (collectively termed as a tunable PDR in Fig. 4) can then optimally balance losses to achieve high transfer fidelity. Multiple emitters may also be implanted ${ }^{21}$ to increase the number of devices containing $\mathrm{SiV}^{-}$ oriented along the applied magnetic field's direction.

In summary, we introduced a phase-stable architecture for highfidelity quantum state transfer between photonic polarization and spin qubits: the fundamental elements of a quantum repeater network. Our simulations and calculations show that the PEPSI can achieve state fidelity exceeding $99 \%$ at kilo-qubits per second transfer rate by carefully balancing losses. We note that the source of infidelity considered in the analyses addresses only the modemismatch error. Namely, the loss imbalance that arises from nonoptimal transmissivities and reflectivities of the PDR and the nanocavity. Additional qubit errors concerning dephasing, spectral diffusion, and charge-state instability will require further investigation $^{22,23}$. Therefore, the calculated fidelity of $99.978 \%$ is realistically an upper bound. Nevertheless, beyond color centers in diamond, our scheme applies to other quantum memories including rare-earth ions ${ }^{24-26}$ and neutral atoms ${ }^{27}$ that may

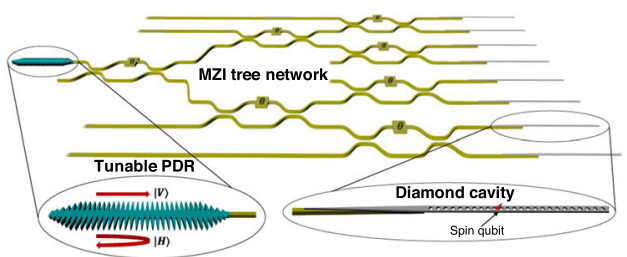

Fig. 4 Implementation in a PIC. A PIC incorporating diamond nanocavities. The $V$ polarization passes through a tunable PDR (a combination of a PDR, active on-chip waveplates and polarizers) and enters an MZI tree network, which routes the photon to an atomcoupled cavity for quantum state transfer. Simulation (see Supplemental Note 7) indicates that an adiabatic taper can provide nearunity coupling efficiency between the PIC and the diamond waveguides. exhibit lower qubit errors. Lastly, we proposed a multiplexing PIC platform for state mappings across a quantum memory array via an MZI tree network. As PIC platforms have scaled beyond tens of individually controllable components ${ }^{28}$, our nanophotonic spinphoton interface should extend these gains to large-scale multiplexed quantum repeaters ${ }^{29}$ and even photonic cluster states $^{30}$.

\section{METHODS}

State transfer fidelity calculations

An incoming qubit is encoded on the polarization of a photon:

$\left|\psi_{P}\right\rangle=a|H\rangle+\beta|V\rangle$.

With the spin qubit prepared in an even superposition state $\left|\psi_{A}\right\rangle=(|\downarrow\rangle+|\uparrow\rangle) / \sqrt{2}$, the joint spin-photon state would be

$\left|\psi_{\text {joint }, i}\right\rangle=a|H, \downarrow\rangle+a|H, \uparrow\rangle+\beta|V, \downarrow\rangle+\beta|V, \uparrow\rangle$.

This photon then hits an imperfect PDR with field reflection (transmission) coefficients $r_{i}\left(t_{i}\right)$ for the polarization $i \in\left\{\begin{array}{l}H, V \\ V\end{array}\right.$. The transmitted output is incident on a nanophotonic cavity coupled to a spin qubit. Since only the $|\downarrow\rangle \Longleftrightarrow\left|\downarrow^{\prime}\right\rangle$ (see Fig. 1a) transition is resonant with the cavity mode, the photon experiences a spin-dependent cavity reflection $r_{\text {cav }} \in\left\{r_{i, \text { uncoupled }}, r_{i, \text { coupled }}\right\}$. The output joint photon-spin system after the HWP (that transform $H \rightarrow H+V, V \rightarrow V-H$ ) is in the state:

$$
\begin{aligned}
\left|\psi_{f}\right\rangle= & |H\rangle \otimes\left[\left(\alpha r_{H, \text { on }}-\beta r_{V, \text { on }}\right)|\downarrow\rangle\right. \\
& \left.+\left(\alpha r_{H, \text { off }}-\beta r_{V, \text { off }}\right)|\uparrow\rangle\right] \\
& +|V\rangle \otimes\left[\left(\alpha r_{H, \text { on }}+\beta r_{V, \text { on }}\right)|\downarrow\rangle\right. \\
& \left.+\left(\alpha r_{H, \text { off }}+\beta r_{V, \text { off }}\right)|\uparrow\rangle\right],
\end{aligned}
$$

where

$$
\begin{aligned}
& r_{H, \text { on }}=r_{H}+\frac{r_{H, \text { coupled }} t_{H}^{2}}{1-r_{H, \text { coupled }} r_{H}}, \\
& r_{H, \text { off }}=r_{H}+\frac{r_{H, \text { uncoupled }} t_{H}^{2}}{1-r_{H, \text { uncoupled }} r_{H}}, \\
& r_{V, \text { on }}=r_{V}+\frac{r_{V, \text { coupled }} t_{V}^{2}}{1-r_{V, \text { coupled }} r_{V}}, \\
& r_{V, \text { off }}=r_{V}+\frac{r_{V, \text { uncoupled }} t_{V}^{2}}{1-r_{V, \text { uncoupled }} r_{V}} .
\end{aligned}
$$

The state-dependent cavity reflection coefficients can be derived from using the input-output formalism ${ }^{31,32}$ :

$$
\begin{aligned}
r(\omega) & =1-\frac{\kappa_{\mathrm{wg}}}{i\left(\omega_{c}-\omega\right)+\frac{\kappa}{2}} \frac{1}{1+\frac{g^{2}}{\left(i\left(\omega_{c}-\omega\right)+\frac{\kappa}{2}\right)\left(i\left(\omega_{a}-\omega\right)+\frac{\gamma}{2}\right)}}, \\
& =\frac{C-1}{C+1}(\text { large C limit }),
\end{aligned}
$$

where $\kappa, \gamma$, and $g$ are the cavity total decay, atom relaxation, and atom-cavity coupling rates. $\kappa_{\mathrm{wg}}$ is the cavity decay rate into the waveguide. $\left(\omega_{c}-\omega\right)$ and $\left(\omega_{a}-\omega\right)$ are the cavity and atom detuning, respectively. $C=\frac{4 g^{2}}{k \gamma}$ is the atom-cavity cooperativity. In the uncoupled case, $g=0$ and a bare reflection off the cavity gives the photon $a-1$ phase. On the other hand, the coupled state results in $a+1$ phase. The relative phase conditioned on the atomic state forms the basis behind the state transfer protocol detailed in ref. ${ }^{3}$.

Finally, a detection of an $|D\rangle$ or $|A\rangle$ photon heralds mapping of the input photonic state onto the spin with an additional Hadamard rotation on the spin (and a conditional $\pi$ rotation). We can calculate the state fidelity by Eq. (1).

Transfer rate calculations

In the state transfer rate calculations, we consider the detection, scattering loss, and error paths for both polarization modes and compute the average rate. Here we consider the transmission efficiency $\eta_{A, V / H}$ through the $H$ attenuator to be $79.8 \%$ for the $V$ polarization and $52.6 \%$ for the $H$ polarization (see Supplementary Note 4 ). We denote the 
power transmission and reflection coefficients of the PDR as $T_{V / H}$ and $R_{V / H}$. The cavity reflectivity $R_{\mathrm{cav}, V} / R_{\mathrm{cav}, H}$ is the average reflectivity between on- and off-resonance cases for the $V / H$ polarization,

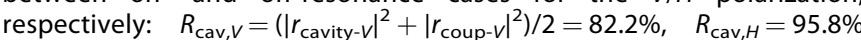
basing on our simulated device and $C=100$.

In order to detect a $V(H)$ photon, it has to either undergo a roundtrip as denoted by Fig. $3 \mathrm{~b}$ with probability $\eta_{\text {link }} \eta_{a, v} T_{v} R_{\text {cav }, V} T_{v} \eta_{\text {det }}\left(\eta_{\text {link }} \eta_{a, H} T_{H} R_{\text {cav }}\right.$ ${ }_{H} T_{H} \eta_{\text {det }}$ ) or reflect off the PDR upon the first pass with probability $\eta_{\text {link }} \eta_{a}$ ${ } R_{v} \eta_{\text {det }}\left(\eta_{\text {link }} \eta_{a, H} R_{H} \eta_{\text {det }}\right)$, where $\eta_{\text {det }}=93.6 \%$ is the detection efficiency accounting for the PBS, the HWP, and the photon detector (see Supplementary Note 4). The average detection probability is then

$p_{\text {det }}=\frac{\eta_{\text {link }}}{2}\left(\eta_{a, V} T_{V}^{2} R_{\text {cav }, V}+R_{V}+\eta_{a, H} T_{H}^{2} R_{\text {cav }, H}+R_{H}\right) \eta_{\text {det }}$.

The photon can also scatter off en route to the PDR with probability $1-$ $\eta_{\text {link, }}$ or by the PDR, contributing to the probability of photon loss without erroring:

$p_{\text {lost }}=1-\eta_{\text {link }}+\eta_{\text {link }}\left(1-\frac{\eta_{\mathrm{a}, \mathrm{V}}+\eta_{\mathrm{a}, \mathrm{H}}}{2}\right)+\frac{\eta_{\text {link }}}{2}\left(\zeta_{\mathrm{V}}+\zeta_{H}\right)$,

where $\zeta_{V}=1-T_{V}-R_{V}$ and $\zeta_{H}=1-T_{H}-R_{H}$.

Lastly, as addressed in the section "Quantum state transfer rate", the photon can be lost after interacting with the atom-cavity system, hence yielding an unheralded error. Specifically, we consider errors arising from the $V$ photon scattering after cavity interaction and the small amount of $H$ photon leaking into the cavity:

$p_{e}=1-p_{\text {det }}-p_{\text {lost }}$.

The probabilities are used to compute $P_{\text {error }}$ which is the probability of at least one unheralded error occurring after detecting the first and only click on the $m$ th attempt within $N$ attempts. After which, we can calculate the average state fidelity:

$\mathcal{F}=\left\langle\psi_{\text {ideal }}\left|\left(1-P_{\text {error }}\right) \rho_{0}+P_{\text {error }} \rho_{\text {mixed }}\right| \psi_{\text {ideal }}\right\rangle$,

where $\rho_{0}$ is the density matrix corresponding to a single attempt considering only device imperfections.

\section{Monte Carlo simulations}

Numerical simulations were performed in MATLAB (MathWorks Inc.). For each attempt, a probability value $p_{\text {random }}$ is chosen out of a uniform distribution $U(0,1) . p_{\text {random }}$ then determines if the photon is lost in the device before reaching the spin $\left(p_{\text {lost }}\right)$, lost in the device after the spin $\left(p_{e}\right)$, or detected $\left(p_{\text {det }}\right)$. Each simulation trial terminates once the photon is detected, and the total experimental time is recorded. A trial can consist of multiple sequences, and each sequence has the number of attempts up to $N_{\max }$, which depends on the fidelity constraint and the link loss. Each Monte Carlo data point presented in Fig. $3 c$ is the average rate of 100 simulation trials.

The oscillation in the Monte Carlo simulations (dashed lines in Fig. $3 c$ ) is an artifact from discretizing the number of attempts required to achieve a certain fidelity constraint given a fixed link loss (see Supplementary Note 4). The allowable $N_{\max }$ is first analytically calculated assuming a continuous variable, then fed into the Monte Carlo simulations to validate the transfer rate calculations. The origin of the artifact can be understood by considering the trade-off between fidelity and the number of attempts. Recall that every undetected photon can be scattered before reaching the emitter-cavity system. Hence, time can be saved by not re-initializing the spin qubit as $N_{\text {attempts }}$ increases. In other words, as link loss increases, it is advantageous to increase the number of attempts. If an integer number of attempts $Z$ is fixed, increasing the link loss consequently lowers the transfer rate until the number of attempts increases to the next integer $Z+1$. After which, the rate increases suddenly and manifests in a sinusoidal behavior in simulations.

\section{DATA AVAILABILITY}

Data available on request from authors.

Received: 13 April 2020; Accepted: 6 November 2020; Published online: 04 January 2021

\section{REFERENCES}

1. Wehner, S., Elkouss, D. \& Hanson, R. Quantum internet: a vision for the road ahead. Science 362, 6412 (2018).

2. Barrett, S. D. \& Kok, P. Efficient high-fidelity quantum computation using matter qubits and linear optics. Phys. Rev. A 71, 060310 (2005).

3. Duan, L.-M. \& Kimble, H. J. Scalable photonic quantum computation through cavity-assisted interactions. Phys. Rev. Lett. 92, 127902 (2004).

4. Tiecke, T. G. et al. Nanophotonic quantum phase switch with a single atom Nature 508, 241-244 (2014).

5. Bhaskar, M. K. et al. Experimental demonstration of memory-enhanced quantum communication. Nature 580, 60-64 (2020).

6. Sukachev, D.-D. et al. Silicon-vacancy spin qubit in diamond: a quantum memory exceeding $10 \mathrm{~ms}$ with single-shot state readout. Phys. Rev. Lett. 119, 223602 (2017).

7. Pingault, B. et al. Coherent control of the silicon-vacancy spin in diamond. Nat. Commun. 8, 15579 (2017).

8. Siyushev, P. et al. Optical and microwave control of germanium-vacancy center spins in diamond. Phys. Rev. B 96, 081201 (2017).

9. Trusheim, M. E. et al. Transform-limited photons from a coherent tin-vacancy spin in diamond. Phys. Rev. Lett. 124, 023602 (2020).

10. Stute, A. et al. Tunable ion-photon entanglement in an optical cavity. Nature $\mathbf{4 8 5}$, 482-485 (2012).

11. Takahashi, H., Kassa, E., Christoforou, C. \& Keller, M. Strong coupling of a single ion to an optical cavity. Phys. Rev. Lett. 124, 013602 (2020).

12. Sun, S., Kim, H., Solomon, G. S. \& Waks, E. A quantum phase switch between a single solid-state spin and a photon. Nat. Nanotechnol. 11, 539-544 (2016).

13. Sun, S., Kim, H., Luo, Z., Solomon, G. S. \& Waks, E. A single-photon switch and transistor enabled by a solid-state quantum memory. Science 361, 6397, 57-60 (2018).

14. Nielsen, M. A. \& Chuang, I. L. Quantum Computation and Quantum Information 10 edn (Cambridge University Press, 2010).

15. Pirandola, S., Laurenza, R., Ottaviani, C. \& Banchi, L. Fundamental limits of repeaterless quantum communications. Nat. Commun. 8, 15043 (2017).

16. Desiatov, B., Shams-Ansari, A., Zhang, M., Wang, C. \& Loncar, M. Ultra-low-loss integrated visible photonics using thin-film lithium niobate. Optica 6, 380-384 (2019).

17. Wan, N. H. et al. Large-scale integration of near-indistinguishable artificial atoms in hybrid photonic circuits. Nature 583, 226-231 (2020).

18. Mouradian, S. L. \& Englund, D. A tunable waveguide-coupled cavity design for scalable interfaces to solid-state quantum emitters. APL Photonics 2, 046103 (2017).

19. Sohn, Y.-I. et al. Controlling the coherence of a diamond spin qubit through its strain environment. Nat. Commun. 9, 2012 (2018).

20. Maity, S. et al. Spectral alignment of single-photon emitters in diamond using strain gradient. Phys. Rev. Appl. 10, 024050 (2018).

21. Evans, R. E. et al. Photon-mediated interactions between quantum emitters in a diamond nanocavity. Science 362, 6415, 662-665 (2018).

22. Grange, T. et al. Cavity-funneled generation of indistinguishable single photons from strongly dissipative quantum emitters. Phys. Rev. Lett. 114, 193601 (2015).

23. Choi, H., Zhu, D., Yoon, Y. \& Englund, D. Cascaded cavities boost the indistinguishability of imperfect quantum emitters. Phys. Rev. Lett. 122, 183602 (2019).

24. Zhong, T., Kindem, J. M., Miyazono, E. \& Faraon, A. Nanophotonic coherent lightmatter interfaces based on rare-earth-doped crystals. Nat. Commun. 6, 8206 (2015).

25. Dibos, A.-M., Raha, M., Phenicie, C.-M. \& Thompson, J.-D. Atomic source of single photons in the telecom band. Phys. Rev. Lett. 120, 243601 (2018).

26. Dutta, S., Goldschmidt, E. A., Barik, S., Saha, U. \& Waks, E. Integrated photonic platform for rare-earth ions in thin film lithium niobate. Nano Lett. 20, 1, 741-747 (2020).

27. Thompson, J. D. et al. Coupling a single trapped atom to a nanoscale optical cavity. Science 340, 6137, 1202-1205 (2013).

28. Qiang, X. et al. Large-scale silicon quantum photonics implementing arbitrary two-qubit processing. Nat. Photonics 12, 534-539 (2018).

29. $\mathrm{Pu}, \mathrm{Y}$.-F. et al. Experimental realization of a multiplexed quantum memory with 225 individually accessible memory cells. Nat. Commun. 8, 15359 (2017).

30. Nemoto, K. et al. Photonic architecture for scalable quantum information processing in diamond. Phys. Rev. X 4, 031022 (2014).

31. Waks, E. \& Vuckovic, J. Dispersive properties and large kerr nonlinearities using dipole-induced transparencyin a single-sided cavity. Phys. Rev. A 73, 041803 (2006).

32. Reiserer, A. \& Rempe, G. Cavity-based quantum networks with single atoms and optical photons. Rev. Mod. Phys. 87, 1379 (2015). 


\section{ACKNOWLEDGEMENTS}

K.C.C. acknowledges funding support by the National Science Foundation Graduate Research Fellowships Program (GRFP), the Army Research Laboratory Center for Distributed Quantum Information (CDQI), and the MITRE Corporation Moonshot program. E.B. is supported by a NASA Space Technology Research Fellowship and the NSF Center for Ultracold Atoms (CUA). D.E. acknowledges support from the NSF EFRIACQUIRE program Scalable Quantum Communications with Error-Corrected Semiconductor Qubits. We also thank Hyeongrak Choi, Ian Christen, and Dr. Matt Trusheim for valuable discussions.

\section{AUTHOR CONTRIBUTIONS}

K.C.C. performed the FDTD simulations in designing the PEPSI. K.C.C. and E.B. calculated the average state fidelity and the transfer rate. D.E. conceived the idea. All authors contributed to writing and revising the manuscript.

\section{COMPETING INTERESTS}

The authors declare no competing interests.

\section{ADDITIONAL INFORMATION}

Supplementary information is available for this paper at https://doi.org/10.1038/ s41534-020-00337-3.
Correspondence and requests for materials should be addressed to K.C.C.

Reprints and permission information is available at http://www.nature.com/ reprints

Publisher's note Springer Nature remains neutral with regard to jurisdictional claims in published maps and institutional affiliations. Attribution 4.0 International License, which permits use, sharing, adaptation, distribution and reproduction in any medium or format, as long as you give appropriate credit to the original author(s) and the source, provide a link to the Creative Commons license, and indicate if changes were made. The images or other third party material in this article are included in the article's Creative Commons license, unless indicated otherwise in a credit line to the material. If material is not included in the article's Creative Commons license and your intended use is not permitted by statutory regulation or exceeds the permitted use, you will need to obtain permission directly from the copyright holder. To view a copy of this license, visit http://creativecommons. org/licenses/by/4.0/.

(c) The Author(s) 2021 